\title{
Poly(methyl metacrylate) Nanocomposites for Two-piece CAD/CAM Solution as an Alternative to Monolithic Removable Prosthesis
}

\begin{abstract}
AIDA PANTAZI ${ }^{1}$, EUGENIA EFTIMIE TOTU2*, DOREL DOROBANTU' ${ }^{1}$, CORINA MARILENA CRISTACHE ${ }^{3 *}$, MARIUS ENACHESCU ${ }^{1}$
'University Politehnica of Bucharest, Center for Surface Science and Nanotechnology (CSSNT), 313 Splaiul Independentei, Bucharest, Romania

2University Politehnica of Bucharest, Faculty of Applied Chemistry and Material Science, 1-5 Polizu Str., 11061, Bucharest, Romania

${ }^{3}$ University of Medicine and Pharmacy Carol Davila, Faculty of Midwifery and Medical Assisting (FMAM),Department of Dental Techniques,8 Eroilor Sanitari Blvd, 050474, Bucharest, Romania

The aim of the present paper was to characterize the new pink PMMA doped with $0.4 \%$ TiO nanoparticles utilized for denture base manufacturing as alternative to the one-piece, single color material'for removable denture. The PMMA base material was structurally characterized through XRD, SEM, EDX, and subsequently byFT-IR and Raman spectroscopy. An improvement in the thermal stability of the obtained material compared with the PMMA matrix without filler was evidenced. All performed structural analyzes are recommending the new pink PMMA with $0.4 \%$ nano-titania as an as an alternative to the one-piece, single color material for removable dentures processing.
\end{abstract}

Keywords: CAD/CAM denture, PMMA nanocomposite, denture base.

The computer-aided design and computer-aided manufacture (CAD/CAM) of removable dentures is less common, comparing to fixed dental restoration, due to the complexity of the technique and data to be integrated, in order to obtain harmonious combination of denture base, artificial teeth, mouth, and face, requiring several recording, transfer, and reevaluation steps [1]. As manufacturing technology, rapid prototyping (RP) has the advantage to reproduce complex shapes, with high accuracy, and reduced material waist, when compared to subtractive technology. Among RP, stereolithography (SLA), based on the use of a UV laser that is vector scanned to photopolymerize the liquid material, creating layer-by-layer the desire shape, with a high spatial resolution (around 50 $\mu \mathrm{m}$ ), was successfully used to obtain poly (methyl methacrylate)(PMMA) complete dentures [2]. The proposed technology allowed obtaining a monolithic denture requiring post processing and esthetic adjustments by applying pink gingiva, light-cured material [2]. In order to avoid increasing thickness of the cameo surface and, consequently, impede on denture fit, a two piece manufacturing solution was envisaged. For the proposed manufacturing technology, two type of photo-cross linkable PMMA was utilized.

The aim of the present paper was to characterize the new pink PMMA doped with TiO, nanoparticles utilized for denture base manufacturing as alternative to the one-piece, single color material for removable denture.

\section{Experimental part}

The 3D printed prostheses manufactured in this study have been obtained using two different types of PMMA composites. For the 3D printed teeth, poly(methylmetharcrylate)(PMMA) filled with metallic oxides, provided by EnvisionTEC (GmbH, Gladbeck, Germany), and reinforced with titania nanoparticles, according to a protocol presented in our previous w orks [2-4], was used. The denture base was obtained from a mixture of PMMA with methylmethacylate (MMA) and a radical reaction promoter (from EnvisionTEC GmbH, Gladbeck, Germany) to which titanium oxide nanoparticles, in both, anatase and rutile, phases (Aldrich), has been added. The complete procedure for obtaining a homogeneous PMMA denture base complies with the preparation method described in detail in another paper [3]. Due to our earlier findings $[2,5,6]$ the optimal amount of the titanium oxide nanoparticles added to the polymeric matrix is $0.4 \%(\mathrm{w} / \mathrm{w})$.

The workflow for two-piece complete denture is similar to the one required for obtaining monolithic removable prosthesis. The final design and the post-processing procedures differ, as could be observed in Figure 1. For two-piece denture manufacturing, two different type of PMMA were utilized. The final prosthesis was assembled using self-curing acrylic resin. For the monolithic Denture, Crea.lign veneering system (Bredent, $\mathrm{GmbH} \&$ Co.KG) was used to obtain pink gingiva on the cameo surface.

The PMMA denture base compositional, structural and morphological properties were characterized using Scanning Electron Microscopy (SEM) and Energy Dispersive $X$-ray Spectroscopy (EDX) techniques. These measurements have been performed on a Hitachi SU 8230 SEM system, equipped with an EDX Oxford detectoranalyzer.

The structural analysis was completed with the results provided by a Perkin Elmer Spectrum Two Fourier Transformed Infrared (FTIR) spectrometer, equipped with an Attenuated Total Reflection (ATR) element. ATR-FTIR spectra have been recorded in $4000-450 \mathrm{~cm}^{-1}$ wavenumber range, with a resolution of $2 \mathrm{~cm}^{-1}$.

The Raman investigations were carried out at room temperature by Confocal Micro-Raman Spectroscopy, using a LabRam HR800 system. All the Raman spectra were generated by exposing the samples during 100 s to a $532 \mathrm{~nm}$ wavelength green excitation laser. The emitted Raman signal of all studied specimens was recorded in $3600-100 \mathrm{~cm}^{-1}$ range, with a spectral resolution of 0.6 $\mathrm{cm}^{-1}$.

The thermal behavior of the denture base PMMA was studied from room temperature $\left(25^{\circ} \mathrm{C}\right)$ up to $1000^{\circ} \mathrm{C}$ using an Universal V4.5A TA Instruments equipment. The applied 

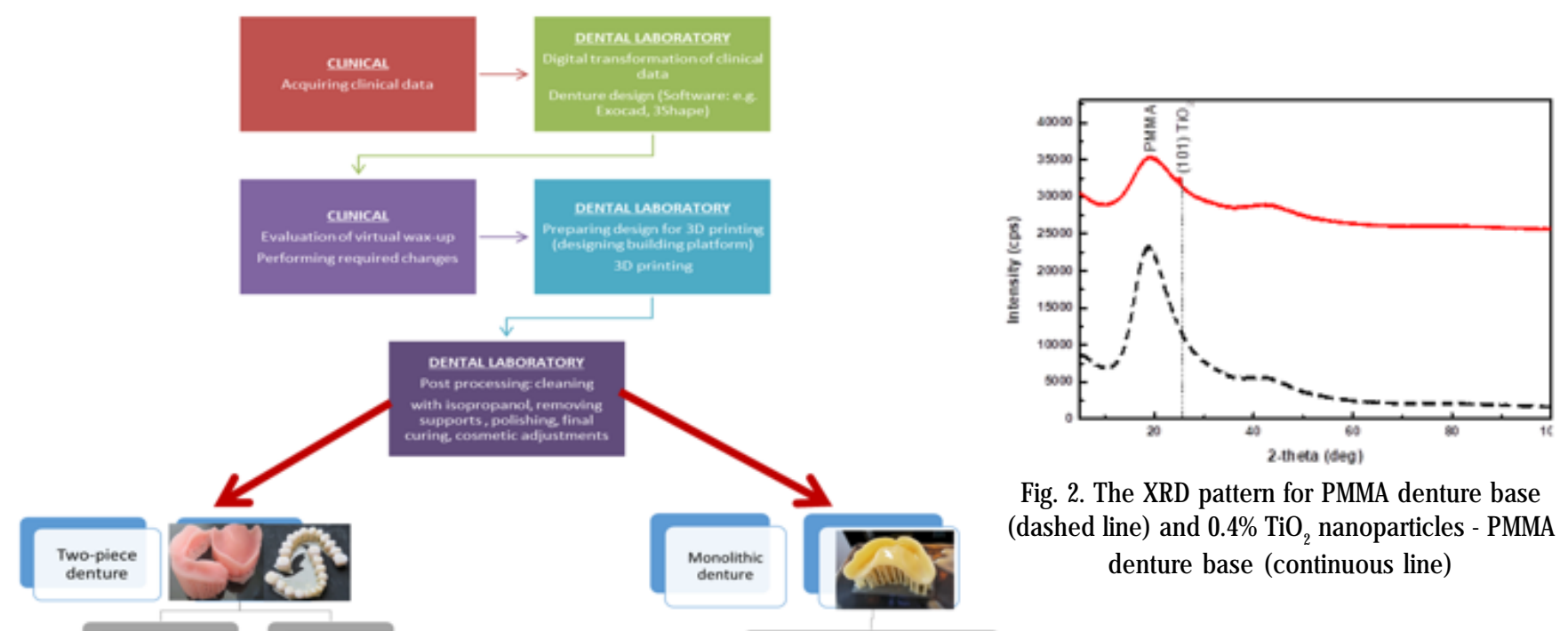

Fig. 2. The XRD pattern for PMMA denture base (dashed line) and $0.4 \% \mathrm{TiO}_{2}$ nanoparticles - PMMA denture base (continuous line)

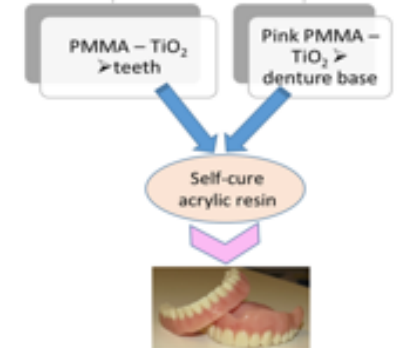

Fig. 1. Workflow scheme for two-piece complete denture and for monolithic removable prosthesis

heating rate was $10^{\circ} \mathrm{C} / \mathrm{min}$ under nitrogen atmosphere (flow rate $50 \mathrm{~mL} / \mathrm{min}$ ). The sample mass was $5 \mathrm{mg}$. The experimental investigations have been performed for the uncured denture base, $0.4 \% \mathrm{TiO}$, nanoparticles - PMMA nanocomposite, as well as for the UV polymerized material resulted from the 3D printer.

The XRD analysis of the prepared $\mathrm{TiO}_{2}$ nanoparticles PMMA denture base sample was performed using a Rigaku SmartLab X Ray Diffractometer, at 9kW (45kV and 200mA) with $\mathrm{Cu} \mathrm{K}_{\alpha}$ radiation: $\mathrm{K}_{\alpha 1}=1.540598 \AA$. The XRD patterns have been recorded within [ $\left.5^{\circ}-100^{\circ}\right] 2 \theta$ range. The phase identification was made by referring to the International Center for Diffraction Data - ICDD (PDF-2) database. The average particle size of the nanoparticles incorporated into the polymeric matrix, the inter-planar spacing between atoms and the specific surface area for TiO nanoparticles (incorporated into the denture base PMMÂ matrix) have been calculated according to the procedure presented in the next section.

\section{Results and discussions}

The X-ray diffraction patterns of PMMA and titania nanoparticles-PMMA nanocomposite denture bases are presented in figure 2.

The $X$-ray diffractograms reveal the XRD characteristic signature of the polymeric matrix - poly (methyl methacrylate) at $2 \theta=17.5^{\circ}$. The weak peak observed in the XRD pattern recorded for the nanocomposite denture base at $2 \theta=22.6^{\circ}$ is corresponding to (101) anatase plane. This maximum is slightly down shifted as it usually appears at $2 \theta=$ $25.3^{\circ}$. The main XRD characteristics observed for PMMA denture base with $0.4 \% \mathrm{TiO}_{2}$ nanoparticles are presented

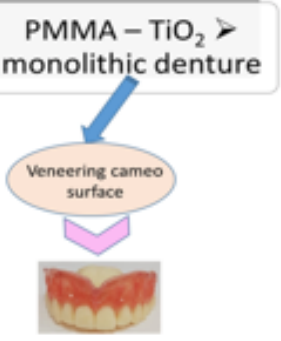

in table 1. The titania nanoparticles dimension was found to be $31 \AA$.

The data presented in table 1, namely the average crystallite size and the inter-planar spacing for each crystallographic orientation have been calculated applying the mathematical relationships based on Bragg diffraction law.

The average nanocrystallite size has been estimated considering Debye-Scherer equation (1):

$$
D=\frac{0.9 \cdot \lambda}{\beta \cdot \cos \theta}
$$

where D stands for crystallite diameter size $(\AA), \lambda=1.5406$ $\AA, \theta$ is the diffraction angle and $\beta$ is the full width at the half maximum in radians (FWHM/rad).

The d-spacing, inter-planar distance, was calculated using the Bragg relationship:

$$
d=\frac{n \cdot \lambda}{2 \cdot \sin \theta}
$$

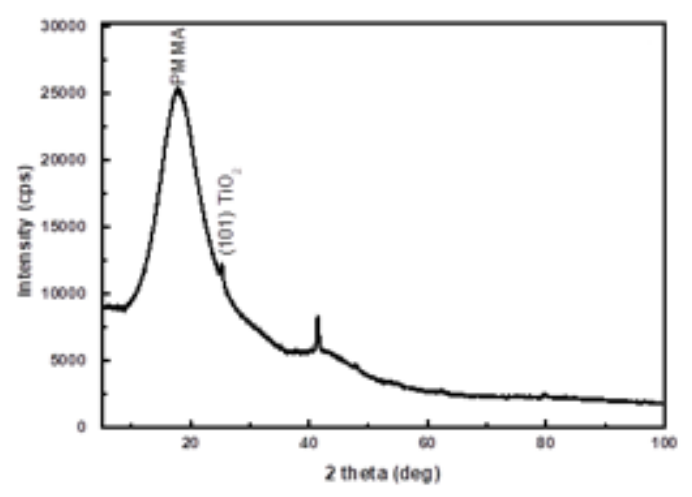

Fig. 3. The XRD pattern of photo-cured PMMA denture base with $\mathrm{TiO}_{2}$ nanoparticles

\begin{tabular}{|c|r|r|r|r|r|r|r|r|}
\hline $2 \theta^{\mathrm{N}}$ & \multicolumn{1}{|c|}{$\theta^{\circ}$} & \multicolumn{1}{c|}{$\sin \theta^{\mathrm{N}}$} & \multicolumn{1}{c}{$\cos \theta^{\mathrm{N}}$} & FWHM( $)$ & FWHM(rad) & $\beta \cos \theta^{\circ}$ & D-Size (A) & d-spacing (A) \\
\hline 17.5049 & 8.7524 & 0.1522 & 0.9883 & 4.2556 & 0.0742 & 0.0733 & 19 & 5.0611 \\
\hline 22.6384 & 11.3192 & 0.1963 & 0.9805 & 2.6464 & 0.0462 & 0.0453 & 31 & 3.9241 \\
\hline 42.8085 & 21.4042 & 0.3649 & 0.9310 & 7.9504 & 0.1387 & 0.1291 & 11 & 2.1110 \\
\hline
\end{tabular}
XRD DATA FOR DENTURE BASE PMMA WITH TIO 2 NANOPARTICLES 


\begin{tabular}{|c|r|r|r|r|r|r|r|r|}
\hline $2 \theta^{\circ}$ & \multicolumn{1}{|c|}{$\theta^{\circ}$} & $\sin \theta^{\circ}$ & \multicolumn{1}{c|}{$\cos \theta^{\circ}$} & FWHM( & FWHM(rad) & $\beta \cos \theta^{\circ}$ & \multicolumn{1}{c}{ Size(A) } & d-spacing (A) \\
\hline 17.9571 & 8.9785 & 0.1560 & 0.9877 & 8.8868 & 0.1551 & 0.1532 & 9 & 4.93574 \\
\hline 25.3285 & 12.6642 & 0.1292 & 0.9756 & 0.4010 & 0.0699 & 0.0682 & 20 & 3.51353 \\
\hline 27.4848 & 13.7424 & 0.2375 & 0.9713 & 5.2475 & 0.0915 & 0.0889 & 16 & 3.24257 \\
\hline 41.4032 & 20.7016 & 0.3535 & 0.9354 & 0.2997 & 0.0052 & 0.0049 & 283 & 2.17905 \\
\hline 45.9104 & 22.9552 & 0.3900 & 0.9208 & 11.8775 & 0.2073 & 0.1909 & 7 & 1.97506 \\
\hline 56.3958 & 28.1979 & 0.4725 & 0.8813 & 10.1178 & 0.1765 & 0.1555 & 9 & 1.63019 \\
\hline 62.1338 & 31.0669 & 0.8840 & 0.8565 & 4.2088 & 0.0734 & 0.0628 & 22 & 1.49271 \\
\hline
\end{tabular}

where $\lambda=1.5406 \AA, \theta$ is the diffraction angle and $d$ represents the d-spacing $(\AA)$.

The diffraction peaks' list revealed by photo-cured $\mathrm{TiO}_{2}$ nanoparticles-PMMA denture base is presented in table $2^{2}$. The parameters shown in table 2 have been calculated applying the equations (1) and (2). The presence of $2 \theta$ peaks at $25.3^{\circ}$ and $45.9^{\circ}$ confirms the successfully incorporation of anatase type titania nanoparticles into the polymer matrix. The broad diffraction peaks from Figure 3 may suggest the presence of significantly small crystallites in material's structure (table 2).

An important physico-chemical characteristic of our material, namely the specific surface area, could be calculated based on the experimental data provided by XRD experiments. The value of the specific surface area allows to determine the specific properties of a particular material. This may be of particular importance to our specific applications for 3D printed denture base fabrication, where surface adsorption is a key factor in the denture quality. The nanocomposite denture base contains titania nanoparticles, therefore nanoparticles' surface state becomes important due to the fact that such nano-oxides have a large surface area related to their volume.

The interaction between the two components of the nanocomposite (PMMA matrix and $\mathrm{TiO}_{2}$ nanoparticles) is expected to be facilitated by the high surface area of the nanoparticles involved. The specific surface area for nanotitania embedded in PMMA matrix was estimated considering the surface area per unit mass, using the following equation:

$$
\text { Specific Surface Area }=\frac{s_{p}}{V_{p} \cdot \rho}
$$

where the particle volume was calculated assuming a spherical shape particle. Therefore, the volume was $V_{p}=$ $4 / 3-\pi-r^{3}$ and the surface area $S_{p}=4-\pi-r^{2}$. The density, $\rho$, of TiO was considered $4.23 \mathrm{~g} / \mathrm{cm}^{3}$.

The specific surface area value calculated for titania nanoparticles (31 $\AA$-table 1 ) embedded in the $4 \% \mathrm{TiO}_{2}$ PMMA nanocomposite (fig. 1), with a volume and a surface area of $15.598 \mathrm{~nm}^{3}$ and $3.019 \mathrm{~nm}^{2}$, respectively, was found to be $45.75 \mathrm{~m}^{2} / \mathrm{g}$ and the $S_{\mathrm{p}} N_{\text {ratio }}$ equal to 0.19 . The ratio between specific surface area and the volume will increase significantly with the decrease of crystallite size. The $\mathrm{TiO}_{2}$ crystallites' average size for the UV cured (UV wavelength ${ }^{2}$ $=340 \mathrm{~nm}$ ) nanocomposite denture base is $20 \mathrm{~nm}$ and, as consequence, the specific surface area value was calculated to be $70.92 \mathrm{~m}^{2} / \mathrm{g}$ and the $S_{p} N_{\text {ratio }} 0.30$.

The FT-IR analysis revealed the two specific peaks of the $-\mathrm{OH}$ group around $3385 \mathrm{~cm}^{-1}$ and $1608 \mathrm{~cm}^{-1}$, corresponding to the stretching and bending vibrations, respectively. The presence of the $-\mathrm{OH}$ functional group is mostlikely due to the adsorbed water molecules. As shown in Figure 4, the presence of the acrylate group within material's structure is confirmed by the appearance of the vibration band around $1716 \mathrm{~cm}^{-1}$, attributed to the $C=0$ stretching mode from acrylate carboxyl group. The vibration bands from $1383 \mathrm{~cm}^{-1}$ and $748 \mathrm{~cm}^{-1}$, known to be $\mathrm{spe}^{-1}$ and $829 \mathrm{~cm}^{-1}$, confirm the presence of poly(methyl methacrylate) in our sample. As it can be easily observed

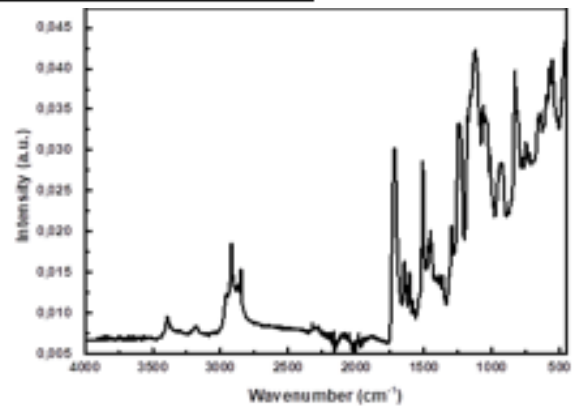

Fig. 4. FTIR spectrum of PMMA denture base with $0.4 \% \mathrm{TiO}_{2}$ composite
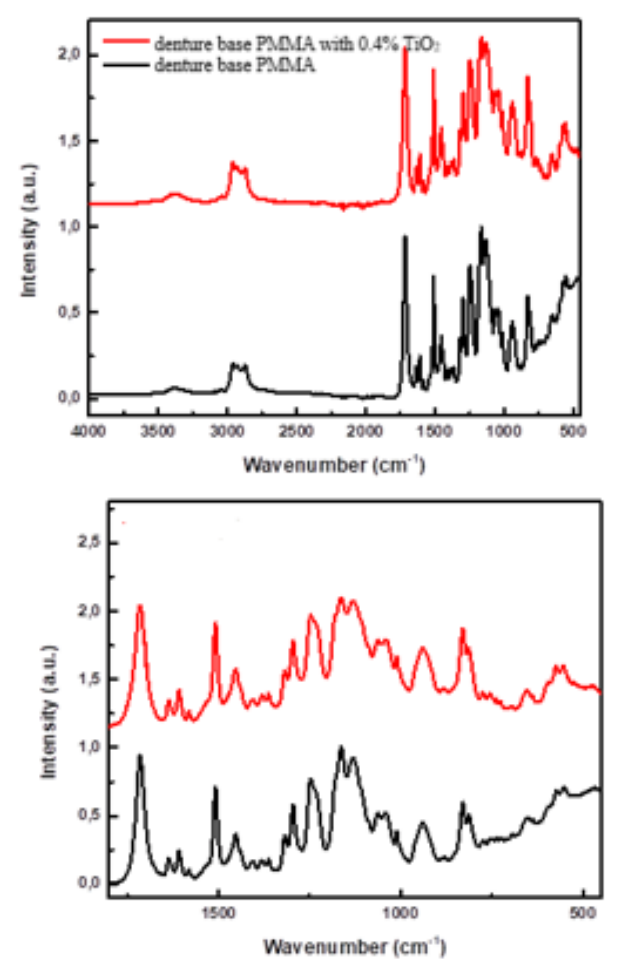

Fig. 5. FTIR spectra for PMMA denture base and PMMA with 0.4\% $\mathrm{TiO}_{2}$ denture base

in figure 4 , the $0.4 \% \mathrm{TiO}_{2}$-PMMA nanocomposite denture base exhibits a fingerprint region characterized by the presence of several peaks. Also, when compared to the FT-IR spectrum recorded for the $0.4 \%$ TiO -PMMA nanocomposite used for $3 \mathrm{D}$ printed teeth [2], a wider band structure in $1200-850 \mathrm{~cm}^{-1}$ spectral range could be observed, which probably covers the characteristic vibrational bands for PMMA: C-O vibration band $f 1161 \mathrm{~cm}$. 1 , and typical absorption bands of PMMA at $1062 \mathrm{~cm}^{-1}$ and $940 \mathrm{~cm}^{-1}$.

In figure 5, FTIR spectra of the PMMA denture base and PMMA with $0.4 \% \mathrm{TiO}_{2}$ nanoparticles denture base were compared to observe the vibrational pattern changes of the nanocomposite spectrum. The band observed at 1400 $\mathrm{cm}^{-1}$ is attributed to Ti-O-Ti vibrational modes and the band from $1645 \mathrm{~cm}^{-1}$ is corresponding to the Ti-OH bending modes $[7,8]$. The FT-IR spectra clearly show that Ti binds to the PMMA matrix by the presence of $\mathrm{Ti}-\mathrm{O}-\mathrm{Ti}$ or $\mathrm{Ti}-\mathrm{OH}$ 


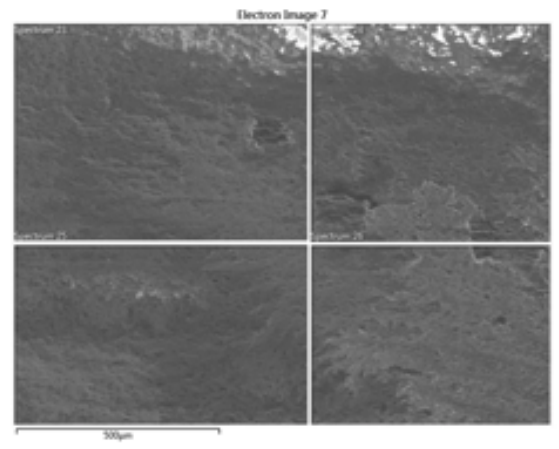

a.

Fig. 6. a. SEM micrographs for PMMA - $0.4 \% \mathrm{TiO}_{2}$ denture base; b. EDX elemental mapping for PMMA $-0.4 \% \mathrm{TiO}_{2}$ denture base

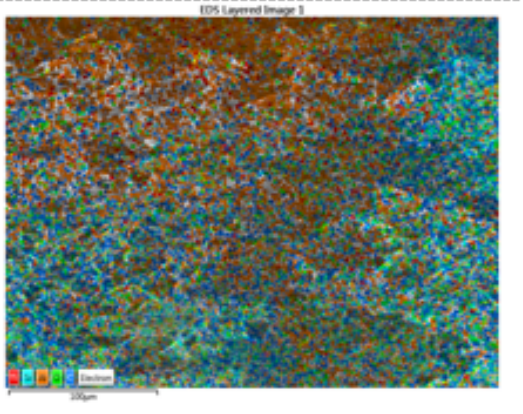

b.
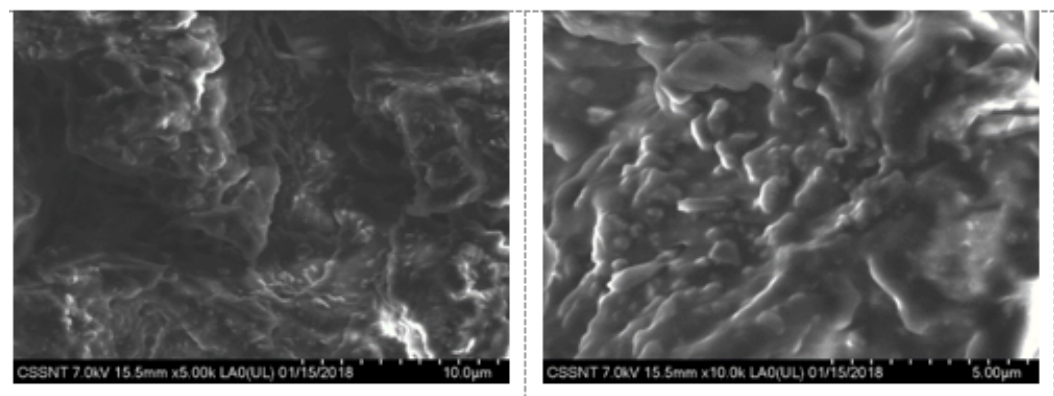

a.

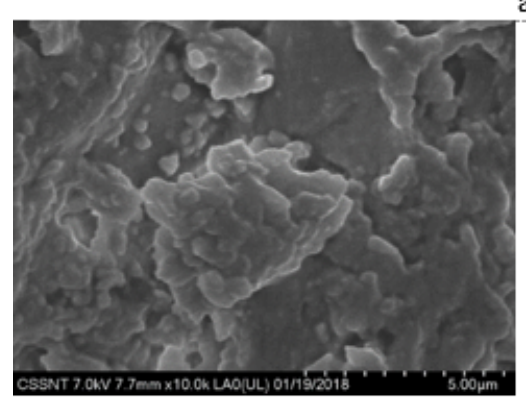

Fig. 7. Low angle backscattered electron images of the

PMMA $-0.4 \% \mathrm{TiO}_{2}$ nanocomposite denture base; a. without Au deposition; b. with Au deposition

bands, resulting into a suitable material for 3D printing technique.

The SEM micrographs of $0.4 \%$ nano-titania - PMMA denture base are presented in figures 6 and 7 . As evidenced in figure 6 , due to their high surface energy the titania nanoparticles have a tendency for agglomeration.

Titanium oxide nanoparticles exhibit good dispersion in the PMMA matrix (fig. 7). SEM analysis has show $n$ that the morphology of the polymeric nanocomposite structure is well arranged and compact. EDX analysis was performed to determine the composition of the sample in different regions of interest.

In figure 8 the Raman spectra recorded for PMMA denture base, $\mathrm{PMMA}$ with $0.4 \% \mathrm{TiO}_{2}$ denture base and $\mathrm{TiO}_{2}$ nanoparticles are presented.

In figure 9 the Raman spectra for both PMMA with $0.4 \%$ $\mathrm{TiO}_{2}$ denture base and $\mathrm{TiO}_{2}$ nanoparticles are illustrated in

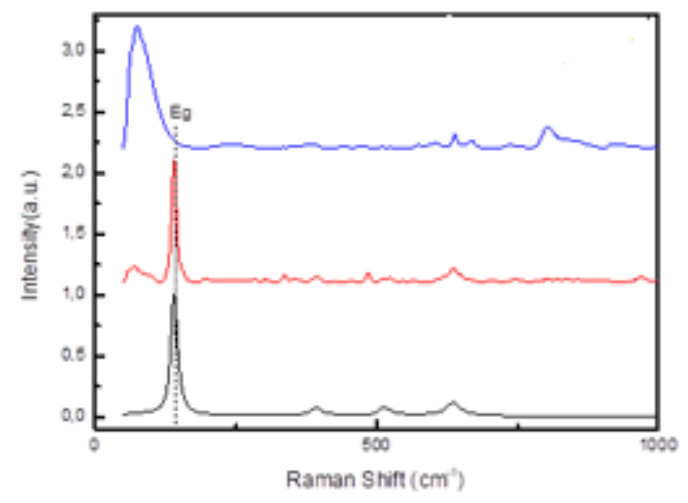

Fig. 8. Comparative Raman spectra for: PMMA denture base (blue line), PMMA with $0.4 \% \mathrm{TiO}_{2}$ denture base (red line), $\mathrm{TiO}_{2}$ nanoparticles (black line) 


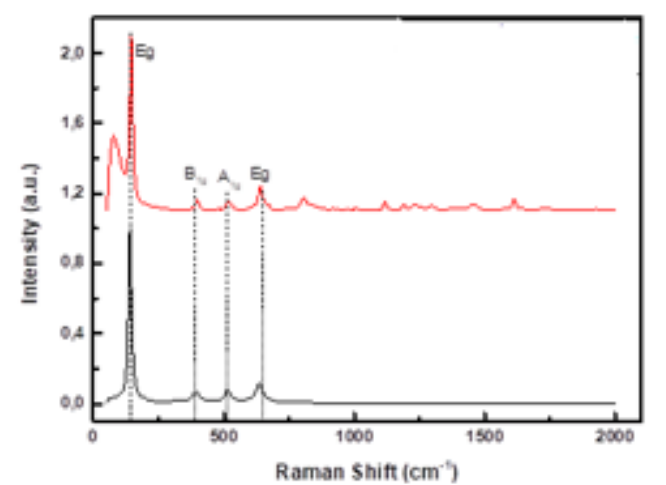

Fig. 9. Comparative Raman spectra for denture base PMMA: denture base PMMA with $0.4 \% \mathrm{TiO}_{2}$ (red line), $\mathrm{TiO}_{2}$ nanoparticles (black line)

order evidence the presence of titania nanoparticles into the polymeric matrix. The Raman frequencies exhibited by the nanocomposite around $398 \mathrm{~cm}^{-1}, 517 \mathrm{~cm}^{-1}$ and 636 $\mathrm{cm}^{-1}$ are assigned to anatase-type titania, while the peaks at $392 \mathrm{~cm}^{-1}$ and $805 \mathrm{~cm}^{-1}$ are attributed to the rutile phase of titania, proving the presence of mixed anatase-rutile titania nanoparticles within the prepared composite.

The result of the thermal analysis is presented in figure 10. Although, the thermogravimetric curve shows a neat and clear step between 100 and $450^{\circ} \mathrm{C}$ with only one obvious decomposition stage, or at most two, actually, the thermogravimetric curve, TGA, presents three major degradation steps which are overlapping. The presence of the three overlapped thermal degradation steps has been evidenced by the first derivative of the thermogravimetric curve (DTG). The linear chain of macromolecular poly (methyl methacrylate) is thermally degraded in four steps (with maximum decomposition rates recorded at: 150, 250,350 and $450 \pm 50^{\circ} \mathrm{C}$ ) marked by huge mass losses.

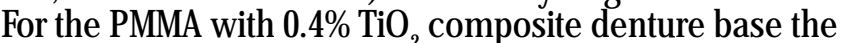
first decomposition step is associated with moisture loss. As known, decomposition temperatures are partially dependent on factors such as type of polymerization, additive presence, type of additive, or filler presence. The analyzed samples are a cross-linked PMMA polymer and PMMA polymer mixed with $0.4 \% \mathrm{TiO}$, nanoparticles, which are degraded in three steps. The first loss is quite small, occurring at temperatures under $100^{\circ} \mathrm{C}$, and it could be assigned to the loss of the moisture as mentioned above. The first decomposition stage that continues up to $200^{\circ} \mathrm{C}$ can be assigned to the volatilization of small molecules/ residual monomers [9]. The second and third steps are described by important mass losses, which may be associated with thermal degradation of various elements of the complex dental base structure and of the polymeric backbone. Taking into account that the thermal decomposition occurred under nitrogen atmosphere, then the formed residues are mainly formed by non-volatile char and minerals.

After the complete thermal decomposition, a very small amount of char and mineral mixture remained in the system. The oxygen species present may interact with the poly (methyl methacrylate) chains resulting in a hydrogen bond or maybe a coordination bond, especially if they are nanostructured (as titania). The direct result of such interaction is an increase in thermal stability of the nanostructured compound. Even without the previously mentioned interaction, there is a possibility that $\mathrm{TiO}_{2}$ nanoparticles will consume a certain amount of heat and consequently increase the thermal stability of the final composite [3].

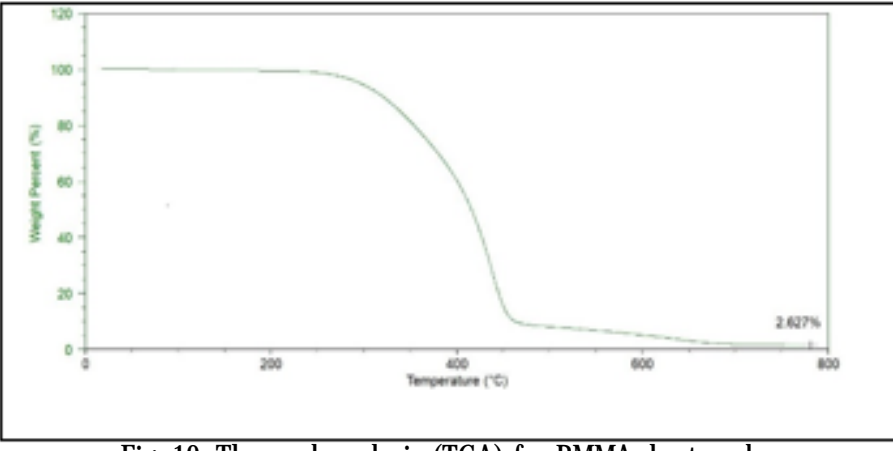

Fig. 10. Thermal analysis (TGA) for PMMA denture base

3D printing technology for complete denture manufacturing was first proposed by Maeda end coworkers in 1994 [10], when the authors used a 3D laser lithography device to additively fabricate shells of removable complete dentures and self-curing acrylic resin was then used to fill the shells and to finally produces the prosthesis. Lately, the commercially available CAD/CAM complete dentures, such as AvaDent digital dentures (Global Dental Science Europe BV, Tilburg, The Netherlands ), were obtained mainly by using subtractive (milling) technology or virtually designed, 3D printed try-in and conventionally processed dentures, such as Dentca CADCAM dentures (DENTCA Inc, Torrance, CA, USA), or Pala Digital Dentures (Heraeus Kulzer, GmbH) [11,12]. Several protocols for interim RP complete dentures have been proposed for reducing production cost [13]. However, studies on the use of CAD/CAM additive technology with photo-cross-linkable polymers for long term removable complete denture manufacturing are scarce. A one-year evaluation of color changes and stainability of ten monolithic 3D printed complete dentures with $0.4 \%$ nanotitania inclusions was performed by our team and the color changes measured over one year were below the maximum acceptability threshold, significantly lower as compared to the same PMMA without TiO [14]. No evaluation was performed on the veneered denture base.

Creating a multicolored prosthesis with a multi-material printing device (using different materials with various properties or color in one printing task) is still a limitation for the printers used in dental laboratories. Therefore, the two-piece complete denture could be a viable, alternative to the monolithic 3D printed prosthesis. Further long-term clinical studies are strongly needed [13].

\section{Conclusions}

The XRD analysis evidenced the presence of nano-titania particles in the PMMA denture base and allowed the calculation of the specific surface area of the material. Also, the morphological characterization showed a homogeneous composite structure, while the Raman and FT-IR investigations proved the good dispersion of $\mathrm{TiO}_{2}$ nanoparticles into the polymer matrix. The $3 \mathrm{D}$ printed composite filled with $0.4 \mathrm{wt}$ \% $\mathrm{TiO}_{2}$ exhibited better thermal stability than the PMMA denture base.

The structural analysis of the new pink PMMA doped with $\mathrm{TiO}_{2}$ nanoparticles recommend the use of this nanocomposite for denture base manufacturing as an alternative to the one-piece, single color material for removable dentures.

Acknowledgement This work was supported by a grant of the Romanian National Authority for Scientific Research and Innovation, CCCDI-UEFISCDI, project number 30/2016 -PRIDENTPRO, (ERA-NETMANUNET II) within PNCDI III. 


\section{References}

1.BIDRA, A., S., TAYLOR, T., D., AGAR, J., R., J. Prosthet. Dent., 109, 2013, p. 361.

2.TOTU, E. E., NECHIFOR, A. C., NECHIFOR, G., ABOUL-ENEIN, H. Y., CRISTACHE, C. M., J. Dent., 59, 2017, p. 68.

3.TOTU, E. E., CRISTACHE, C. M., VOICILA, E., OPREA, O., AGIR, I., TAVUKCUOGLU, O., DIDILESCU, A. C., Mat. Plast., 54, no. 4, 2017, p.666.

4.CRISTACHE, C., M., TOTU, E., E., MOCANU, G., BUTNARASU, C., DentalTarget., vol. XI, no. 3(4), 2016, p. 32.

5.TOTU, E. E., VOICILA, E., PISTRITU, V., NECHIFOR, G., CRISTACHE, C. M., Rev. Chim.(Bucharest), 69, no.1, 2018, p. 155.

6.TOTU, E. E., CRISTACHE, C. M., ISILDAK, I., YILDIRIM, R., BURLIBASA, M., NIGDE, M., BURLIBASA, L., Rev. Chim.(Bucharest), 69, no.5, 2018, p.1160.

7.HERRERA-SANDOVAL, G., BAEZ-ANGARITA, D., CORREA-TORRES, S., PRIMERA-PEDROZO, O., HERNANDEZ-RIVERA, S., Materials Sciences and Applications, 4, no.3, 2013, p. 179.
8.CAO, J., WANG, A.,L., HE, X., FANG, M., GAO, J., LI, J. , DENG, L., CHEN, H., TIAN, G., WANGA, J., FANE, S., J. Mater. Chem. A, 1, 2013, p. 5955.

9.TOTU, E.E., Rev.Chim. (Bucharest), 57, no. 9, 2006, p.963-966. 10.MAEDA, Y., MINOURA, M., TSUTSUMI, S., OKADA, M., NOKUBI, T., Int. J. Prosthodont., 7, 1994, p.17.

11.KATTADIYIL, M., T., ALHELAL, A., J. Prosthet. Dent., 117, 2016, p.478.

12.HAN, W., LI, Y., ZHANG, Y., LV, Y., ZHANG, Y., HU, P., LIU, H., MA, Z., SHEN, Y., Med. (United States), 96, 2017, p. e5435.

13.LIN, W. S., HARRIS, B. T., PELLERITO, J., MORTON, D., J. Prosthet. Dent., 2018, doi:10.1016/j.prosdent.2017.12.027, in press.

14.CRISTACHE, C., M., OANCEA, L., DIDILESCU, A.,C., BURLIBASA, M., TOTU, E., E., Rev. Chim.(Bucharest), 69, no.2, 2018, p. 463.

Manuscript received:11.09.2018 